\begin{tabular}{|c|c|}
\hline Citation/Reference & $\begin{array}{l}\text { Matic V., Cherian P.J., Koolen N., Naulaers G., Swarte R.M., Govaert P., Van } \\
\text { Huffel S., De Vos M., " } \text { Holistic approach for automated background EEG } \\
\text { assessment in asphyxiated full-term infants", Journal of neural engineering, } \\
\text { vol. 11, no. 6, Oct. 2014, pp. 1-14. }\end{array}$ \\
\hline Archived version & Final publisher's version / pdf \\
\hline Published version & $\begin{array}{l}\text { insert link to the published version of your paper } \\
\text { http://dx.doi.org/10.1088/1741-2560/11/6/066007 }\end{array}$ \\
\hline Journal homepage & $\begin{array}{l}\text { insert link to the journal homepage of your paper } \\
\text { http://iopscience.iop.org/1741-2552/. }\end{array}$ \\
\hline Author contact & Vladimir.matic@esat.kuleuven.be \\
\hline IR & url in Lirias https://lirias.kuleuven.be/handle/123456789/477336 \\
\hline
\end{tabular}


Holistic approach for automated background EEG assessment in asphyxiated full-term infants

This content has been downloaded from IOPscience. Please scroll down to see the full text.

2014 J. Neural Eng. 11066007

(http://iopscience.iop.org/1741-2552/11/6/066007)

View the table of contents for this issue, or go to the journal homepage for more

Download details:

This content was downloaded by: maticvl

IP Address: 134.58.253.57

This content was downloaded on 26/02/2015 at 11:07

Please note that terms and conditions apply. 


\title{
Holistic approach for automated background EEG assessment in asphyxiated full-term infants
}

\author{
Vladimir Matic $^{1,2}$, Perumpillichira J Cherian ${ }^{3}$, Ninah Koolen ${ }^{1,2}$, \\ Gunnar Naulaers ${ }^{4}$, Renate M Swarte ${ }^{5}$, Paul Govaert ${ }^{5}$, \\ Sabine Van Huffel ${ }^{1,2}$ and Maarten De Vos ${ }^{6}$
}

${ }^{1}$ KU Leuven, Department of Electrical Engineering (ESAT) STADIUS Centre for Dynamical Systems, Signal Processing and Data Analytics, Leuven, Belgium

${ }^{2}$ iMinds Medical IT Department, Leuven, Belgium

${ }^{3}$ Section of Clinical Neurophysiology, Department of Neurology, Erasmus MC, University Medical Center, Rotterdam, The Netherlands

${ }^{4}$ Neonatal Intensive Care Unit, University Hospital Gasthuisberg, Leuven, Belgium.

${ }^{5}$ Section of Neonatology, Department of Pediatrics, Erasmus MC-Sophia Children's Hospital, University Medical Center, Rotterdam, The Netherlands

${ }^{6}$ Institute of Biomedical Engineering, Department of Engineering, University of Oxford, Oxford, UK

E-mail: Vladimir.Matic@esat.kuleuven.be

Received 21 May 2014, revised 27 August 2014

Accepted for publication 19 September 2014

Published 31 October 2014

\begin{abstract}
Objective. To develop an automated algorithm to quantify background EEG abnormalities in full-term neonates with hypoxic ischemic encephalopathy. Approach. The algorithm classifies $1 \mathrm{~h}$ of continuous neonatal EEG (cEEG) into a mild, moderate or severe background abnormality grade. These classes are well established in the literature and a clinical neurophysiologist labeled $2721 \mathrm{~h}$ cEEG epochs selected from 34 neonates. The algorithm is based on adaptive EEG segmentation and mapping of the segments into the so-called segments' feature space. Three features are suggested and further processing is obtained using a discretized threedimensional distribution of the segments' features represented as a 3-way data tensor. Further classification has been achieved using recently developed tensor decomposition/ classification methods that reduce the size of the model and extract a significant and discriminative set of features. Main results. Effective parameterization of cEEG data has been achieved resulting in high classification accuracy (89\%) to grade background EEG abnormalities. Significance. For the first time, the algorithm for the background EEG assessment has been validated on an extensive dataset which contained major artifacts and epileptic seizures. The demonstrated high robustness, while processing real-case EEGs, suggests that the algorithm can be used as an assistive tool to monitor the severity of hypoxic insults in newborns.
\end{abstract}

Keywords: background EEG classification, perinatal asphyxia, tensor classification, multi-linear algebra, EEG quantification

\section{Introduction}

Perinatal hypoxic ischemic encephalopathy (HIE) represents a major factor that cause morbidity and mortality in newborns (Kinoti 1993, Hagberg et al 2001, Richardus et al 2003). In survivors, the common sequelae include cerebral palsy, epilepsy and/or cognitive problems. The injury is mainly determined by the severity and duration of the insult (Richardus et al 2003). Subsequently, in Neonatal Intensive Care Units (NICUs), clinicians need to establish a prompt diagnosis of 
the initial degree of the hypoxic insult, monitor the progress of the injury and make treatment decisions. For this purpose, several monitoring utilities are used as a standard clinical practice in NICUs. For instance, the clinical Thompson and Sarnat scores are used to assess the degree of HIE severity. In addition, amplitude integrated electroencephalogram (aEEG) is commonly used to monitor dynamic changes in brain functioning. aEEG has proved to have a high sensitivity and specificity to predict outcome within $6 \mathrm{~h}$ after birth (De Vries and Hellström-Westas 2005). However, this is in the hands of experienced readers and often extra EEG validation is needed. Hence, a more suitable bed-side monitoring utility to study brain function in detail is full continuous EEG (cEEG) signal acquired with 17 (or 13) electrodes. However, visual interpretation of the EEG signal is laborious and requires a high level of expertise. Moreover, the presence of expert EEG readers is not always available and, in particular, smaller medical centers usually lack this support. As a result, critically ill newborn babies may receive sub-optimal treatment. On the other hand, the current situation can be improved with an automated EEG analysis system that would provide continuous real-time decision support to the clinical staff. The system needs to monitor and assess recovery of background EEG activity. For this purpose, the severity of background EEG abnormality is commonly assessed at 12, 24, 36, 48 and $72 \mathrm{~h}$ post partum (Murray et al 2009). This would provide an insight into treatment response and parameters for the assessment of neurodevelopmental outcome.

A visual interpretation of EEG signals is used as the 'gold' standard in clinical practice, whereas automated EEG analysis has been mainly a research tool. For instance, automated EEG segmentation algorithms have been previously developed (e.g. Barlow et al 1981, Krajca et al 1991). More specifically, in term and premature newborns, research has focused on analysis of certain EEG patterns. For example, effective sleep-wake cycle detection methods (Scher 2004, Gerla et al 2009), characterization of neonatal sleep EEG patterns (Barlow 1985, Paul et al 2003), localization of ictal activity (Despotovic et al 2013) and automated neonatal seizure detectors (Gotman et al 1997, Deburchgraeve et al 2008, Temko et al 2011, Cherian et al 2011) have been proposed.

Few results are, however, published to automatically assess background EEG abnormality of full-term neonates with HIE. In general, two different approaches are presented in the literature. On the one hand, some approaches focus on the detection of EEG patterns for visual grading of EEG abnormalities. For instance, algorithms for the detection of bursts or interburst intervals (IBIs) are developed (Löfhede et al 2008, Matic et al 2012, 2014). Several studies, that analyzed premature neonates, also describe algorithms for EEG burst/suppression pattern detection (Palmu et al 2010, Jennekens et al 2011, Mitchell et al 2013, Koolen et al 2014). On the other hand, researchers have developed methods to detect background EEG states as assessed by an expert EEG reader from selected EEG segments with a duration of $1 \mathrm{~h}$. For instance, a method proposed in Löfhede et al 2010 detects 'burst-suppression' state to distinguish neonates with severe
HIE from healthy newborns. Furthermore, methods for automated scoring of background EEG abnormality are proposed in (Korotchikova et al 2011, Stevenson et al 2013). These methods consist of a proposed set of discriminative quantitative EEG (qEEG) features, which are used as input into machine learning classifiers. Subsequently, classifiers are optimized to replicate decisions brought by expert EEG readers.

However, in the quest to develop fully automated EEG analysis systems researchers encounter several bottlenecks. First, the number of neonates in datasets is relatively small for the development of inductive (supervised) learning methods. In some studies only six (Löfhede et al 2008) or 13 neonates (Paul et al 2003) were used. Larger studies included datasets from only 20-54 neonates (Löfhede et al 2010, Korotchikova et al 2011). Hence, low sample size make the use of machine learning techniques challenging. Second, short EEG epochs are analyzed per neonate in order to derive conclusions. For instance, $10 \mathrm{~min}$ epochs were selected and analyzed per neonate in (Paul et al 2003), whereas 7 min epochs were selected per neonate and used for training of inductive classifiers by Stevenson et al 2013. This is rather restrictive for the analysis of the EEG signal that is very nonstationary and can rapidly evolve over time. Third, artifacts represent most likely the major bottleneck for reliable automated EEG analysis. To avoid deterioration of performance, researchers usually preselect segments without artifacts and seizures and derive sets of discriminative qEEG features for background EEG state classification (Paul et al 2003, Korotchikova et al 2011, Stevenson et al 2013).

To develop robust and reliable automated EEG analysis systems significant progress has been achieved with EEG artifact-removal tools. For example, methods for removing muscle, electrocardiogram, pulsation and respiration artifacts have been published (e.g. Joyce et al 2004, Klemm et al 2009, De Vos et al 2010, 2011). However, these methods are mostly tuned for one or a few specific artifacts, whereas in fully automated analysis of EEG background, a diverse range of artifacts are encountered that adversely affects system's performance. For example, electrode and wire problems, movement and transient muscle artifacts, to name a few, will hinder the detection accuracy. Unfortunately, a majority of artifacts as well as electrographic seizures are superimposed onto suppressed and discontinuous EEG periods, thereby masking discontinuity and hindering its assessment. Therefore, the presence of artifacts might bias the decisions of automated methods from severe abnormalities (longer EEG discontinuities) towards the group of milder EEG abnormalities.

In this study we present a method for automated assessment of background EEG abnormalities in neonates with HIE, that strives to tackle the major bottlenecks of current approaches. It is our objective to classify cEEG epochs according to one of the three severity grades (mild, moderate or severe) as would have been scored by an experienced clinical neurophysiologist without preselecting clean data segments and including several evolving cEEG epochs per neonate. We propose a tensor-based classification method that 


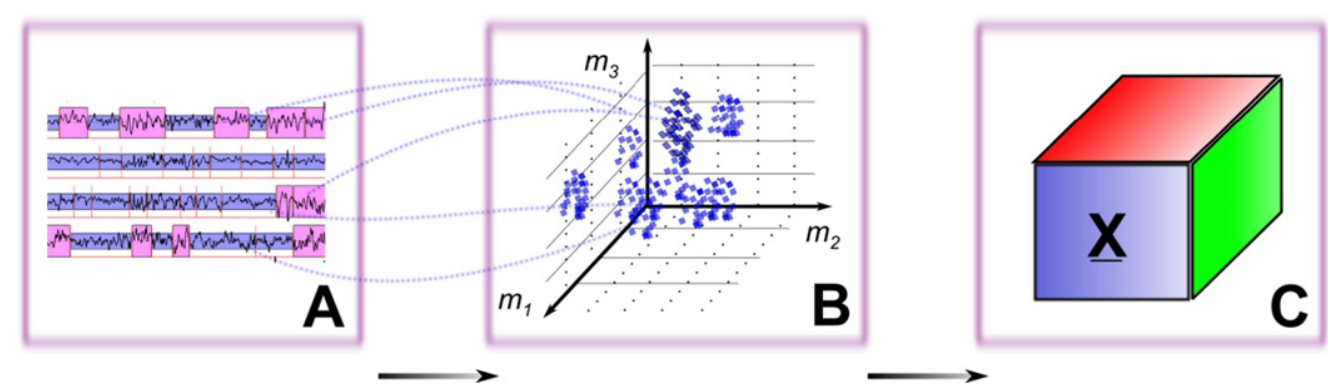

Figure 1. Three blocks represent the parameterization of the cEEG data stream. (A) First, the EEG signal is adaptively segmented. (B) Segment's features are calculated for each segment and depending on the quantized features' index values $\left(m_{1}, m_{2}, m_{3}\right)$ they are mapped into the discretized segments' feature space. (C) 3D distribution is parameterized using the tensor representation to effectively capture the structure of the distribution. That is, tensor elements represent prevalence of segments with a particular discretized feature distribution.

can cope with the low sample size and the presence of moderate disturbing EEG segments. The performance and robustness of the proposed method will be assessed as on a dataset of 34 newborns who have suffered perinatal asphyxia.

\section{Methods}

\subsection{Dataset}

EEG data have been recorded at Sophia Children's Hospital, Erasmus MC (Rotterdam, The Netherlands). The 34 neonates included in this study were part of a larger cohort of term asphyxiated neonates who were monitored from March 2003 till August 2007. The inclusion criteria for EEG monitoring were: gestational age of 37-43 weeks with clinical features of encephalopathy, and having at least one of the following features of birth asphyxia: (a) arterial $\mathrm{pH}$ of umbilical cord blood $\leqslant 7.1$, (b) Apgar score $\leqslant 5$ at $5 \mathrm{~min}$, and (c) high clinical suspicion (like fetal distress, umbilical cord prolapse, difficult labor, or a history of convulsions). Babies with congenital cardiac abnormalities, multiple congenital anomalies and inborn errors of metabolism were excluded. In addition, the neonates expressing constantly low voltage inactivity $(<5 \mu \mathrm{V})$, that is, isoelectric background EEG activity, were excluded as well. The study had the approval of the Erasmus MC Medical Ethical Review Board. Informed consent was obtained from the parents/guardians prior to the onset of the registration. The EEG recordings started 2-48 (median 19) hours post partum. The median (range) duration of cEEG was 31 (20-96) hours. Seizures were recorded in 18 patients and were treated according to a protocol with anti-epileptic drugs. The applied EEG sampling frequency was $256 \mathrm{~Hz}$ and the EEG data were analyzed using bipolar montage with 12 channels. The data have been filtered using standard FIR high-pass and low-pass filters at $0.7 \mathrm{~Hz}$ and $20 \mathrm{~Hz}$.

In this study, four epochs of $2 \mathrm{~h}$ continuous EEG recordings have been selected per neonate. The epochs were selected equidistantly across EEG recordings such that the minimal distance between $2 \mathrm{~h}$ epochs is longer than $4 \mathrm{~h}$. Every hour of the $2 \mathrm{~h}$ segments was independently graded, making in total $272(=34 \times 4 \times 2) 1 \mathrm{~h} \mathrm{cEEG}$ epochs. An experienced clinical neurophysiologist (PJC), blinded to the clinical details of the neonates, visually assessed background EEG abnormalities and classified them into: mild (and normal), moderate and severe classes. The applied background EEG grading system was adapted from the one proposed in (Murray et al 2009) resulting in:

(a) mild (and normal)—approximately IBIs $<5 \mathrm{~s}$; poorly organized sleep-wake cycle; recovered, continuous background EEG is assigned to this class as well

(b) moderate-approximately $5 \mathrm{~s} \leqslant$ IBIs $<10 \mathrm{~s}$

(c) severe abnormality- $10 \mathrm{~s} \leqslant$ IBIs $<60 \mathrm{~s}$, characterized by prolonged and marked suppressions.

\subsection{Multi-dimensional structure for capturing EEG dynamics}

Tensor methods provide a powerful tool to preserve underlying multidimensional structure and have been shown to be useful to analyze EEG data (De Vos et al 2007, Vanderperren et al 2013). We will exploit the tensor structure of background EEG as illustrated in figure 1. First, EEG channels are independently segmented using an adaptive segmentation algorithm (Krajca et al 1991). Next, three features (see section 2.3) are computed for each segment. These features can be discretized into particular bins and prevalence in the different bins can be quantified as a histogram. The resulting tensor represents the prevalence of segments with a particular segments' feature distribution (figure 1).

In the literature, it has been shown that similar underlying brain functioning generate similar distributions of segments' features (Bodenstein et al 1985, Creutzfeldt et al 1985). That is, two EEG data streams showing similar patterns (e.g. tracé alternant sleep state in newborns) are expected to generate similar distributions in the segments' feature space (Van Hese et al 2001). Therefore, we assume that similar background EEG abnormalities also produce similar distributions in the segments' feature space.

\subsection{Design of segments' features}

To obtain an automated assessment of the acute severity of neonatal brain injury, features used for the parameterization of EEG segments should be correlated with the expert visual grading of background EEG abnormalities. For example, distribution of EEG amplitude represents a distinctive 
Table 1. Index feature $m_{1}$ obtains values according to the corresponding amplitudes' ranges.

\begin{tabular}{lccccccccc}
\hline Amplitude $(\mu \mathrm{V})$ & $0-5$ & $5-10$ & $10-20$ & $20-30$ & $30-40$ & $40-50$ & $50-60$ & $60-120$ & $>120$ \\
\hline$m_{1}$ & 1 & 2 & 3 & 4 & 5 & 6 & 7 & 8 & 9 \\
\hline
\end{tabular}

hallmark used by experts to classify EEG background (Watanabe et al 1980, Murray et al 2009). EEG amplitude is expected to have higher values in mild background EEG abnormalities and in continuous EEG periods. In addition, spatial (global) attenuation of amplitude is pronounced in severe HIE, when EEG becomes more simplistic and suppressed. Moreover, the interplay between adjacent segments with different amplitudes within single EEG channel can be used to capture variability of the EEG signal. For instance, higher amplitude variability is expected in more continuous EEG periods.

Here, the following features are defined to characterize background EEG abnormalities. For each EEG segment we quantize features' values and calculate three indexes $m_{1}, m_{2}$ and $m_{3}$, which would map an EEG segment into the corresponding distribution tensor element $\underline{\mathbf{X}}\left(m_{1}, m_{2}, m_{3}\right)$. In this way, a three-dimensional histogram tensor $\underline{\mathbf{X}}$ would parameterize overall cEEG epoch. According to the visual interpretations and guiding rules for the background EEG grading, we suggest the range of features' values and the quantized values of indexes as follows:

$m_{1}$ [1-9]: peak-to-peak amplitude is calculated independently for every EEG segment. According to the peak-to-peak amplitude values $m_{1}$ obtains the following index values as suggested in table 1 .

$m_{2}$ [1-10]: represents global, spatial amplitude of the EEG data. At the time point, exactly at the segment's center, the amplitude of the spatially aligned segments is estimated across all EEG channels as follows. First, the EEG signal is independently segmented and the segments are classified according to their amplitude values into: Low $(L)<20 \mu \mathrm{V}$, Medium $(M)<40 \mu \mathrm{V}$ and High $(H) \geqslant 40 \mu \mathrm{V}$. Next, we count for each class the occurrences of these segments across EEG channels. To accomplish this we use three temporal profile signals one for each amplitude class: Low (LTP), Medium (MTP) and High (HTP). The creation of temporal profile signals is depicted in the figures 2(A) and (B). Subsequently, from the temporal profile signals we derive the $m_{2}$ index:

$$
\begin{aligned}
m_{2}= & \text { floor }((\text { LTP }+ \text { MTP } \cdot 1.5+\text { HTP } \cdot 2) \\
& -12) / 12 \cdot 10)+1,
\end{aligned}
$$

floor-rounds towards negative infinity.

The segment's index value $\left(m_{2}\right)$ is in the range from 1 to 10 (figure 2(C)). Value 12 corresponds to the number of EEG channels, whereas weighting factors are set to 1.5 and 2 . Lower $m_{2}$ index values, $m_{2} \in[1-2]$, determine the suppressed EEG periods (e.g. from the 7th till the 13th second in the figures 2(A) and (B)). On the other hand, continuous EEG patterns commonly have $m_{2} \in[5-7]$, whereas burst periods are characterized with values $m_{2} \in[8-10]$.
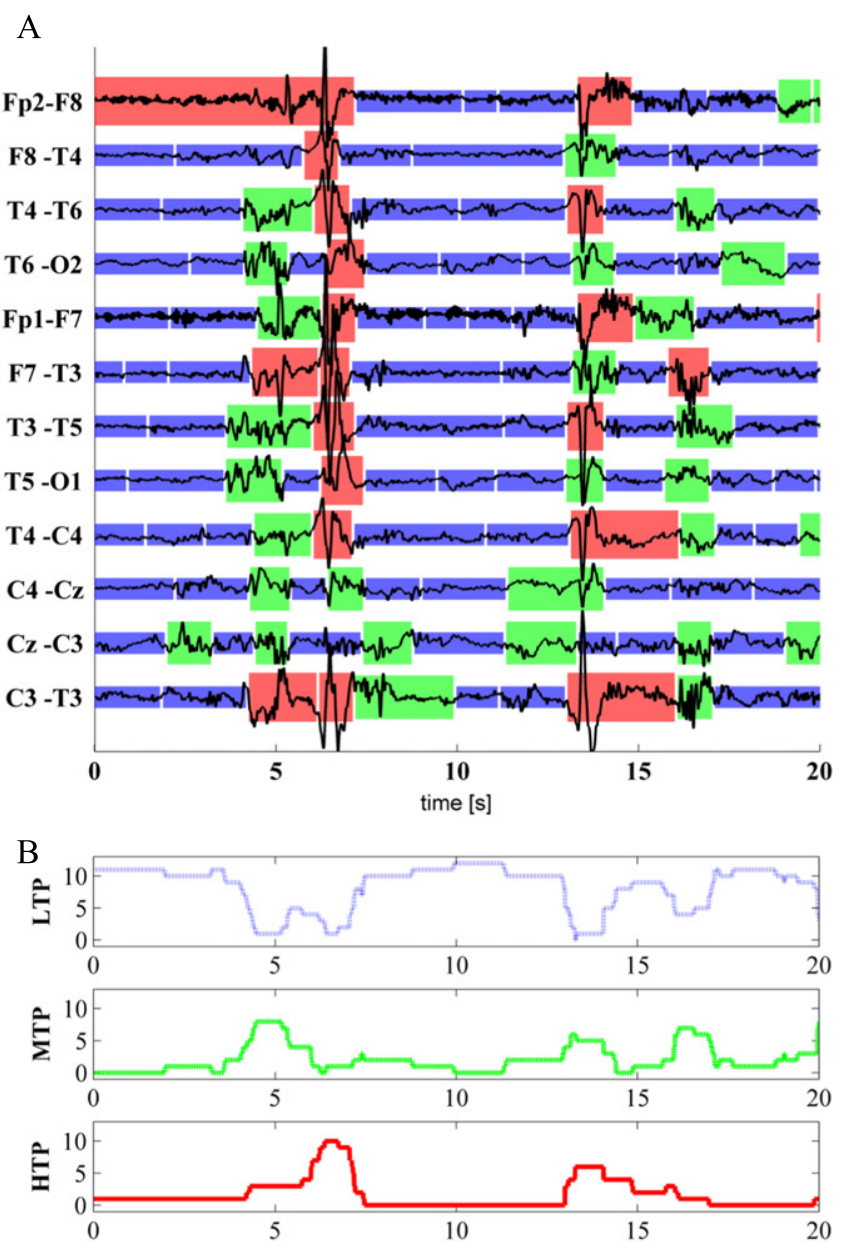

C

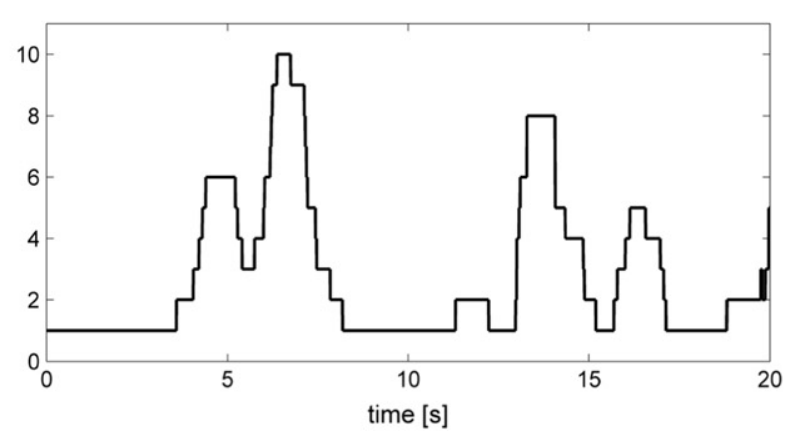

Figure 2. (A) An EEG epoch is adaptively segmented and the segments are classified into three peak-to-peak amplitude classes: Low $<20 \mu \mathrm{V}$ (blue), Medium $<40 \mu \mathrm{V}$ (green) and High $\geqslant 40 \mu \mathrm{V}$ (red). (B) Three temporal profile signals (LTP, MTP and HTP) count the occurrences of the amplitude classes across EEG channels. (C) Global grade of the spatial EEG amplitude, $m_{2}$ value, is derived using the signals (LTP, MTP and HTP) as follows: $m_{2}=$ floor $(((\mathrm{LTP}+\mathrm{MTP} \cdot 1.5+\mathrm{HTP} \cdot 2)-12) / 12 \cdot 10)+1$.

$m_{3}$ [1-12]: represents the duration of the consecutive segments having the same amplitude class $(L, M$ or $H)$ within the single EEG montage channel. First, we determine the 
Table 2. A segment is classified into one of the three amplitude classes: Low $(L)$, Medium $(M)$ or High $(H)$. Within the single EEG channel, adjacent segments that express the same amplitude class are merged and duration of this interval is calculated. The interval duration determines the values for $m_{3}$ index parameter according to the table. All consecutive segments which are merged in this way would have the same $m_{3}$ index value regardless of their position in this segments' stream.

\begin{tabular}{lccr}
\hline$m_{3}$ index value interval (s) & Low & Medium & High \\
\hline $0-5$ & 1 & 5 & 9 \\
$5-10$ & 2 & 6 & 10 \\
$10-20$ & 3 & 7 & 11 \\
$>20$ & 4 & 8 & 12 \\
\hline
\end{tabular}

amplitude class of a segment: Low, Medium or High. Next, we calculate the maximum duration of the adjacent segments having the same amplitude class. Finally, the quantized index value $m_{3}$ would have the following values as suggested in table 2 .

The following example illustrates the ability of segments' features to capture various interplays of the adjacent EEG segments. Five segments (A, B, C, D, E) are selected and indices $m_{1}, m_{2}$ and $m_{3}$ are calculated (figure 3(A)). For example, segments $\mathrm{A}$ and $\mathrm{E}$ are assigned to frequency bin (2, $4,1)$ since their amplitude is attenuated, they are spatially aligned with segments that do not represent the global EEG attenuation $\left(m_{2}=4\right)$ and they are isolated low amplitude class segments $\left(m_{3}=1\right)$. Segment B is mapped into $(7,5,9)$ as it is a high amplitude segment, spatially aligned with segments of 'normal' EEG variability and merged with the consecutive high class segment the entire interval duration is shorter than $5 \mathrm{~s}\left(m_{3}=9\right)$. Segment $\mathrm{C}$, mapped into $(2,6,4)$, is a low amplitude segment quantifying prolonged suppressed activity in this EEG channel ( $>20 \mathrm{~s}$ ) and therefore $m_{3}=4$. On the other hand, the segment D corresponds to the frequency bin $(4,2$, 6 ), since it is a medium class segment that together with the neighboring medium class segments encompasses an interval from 5 till $10 \mathrm{~s}\left(m_{3}=6\right)$.

Further illustration of mapping the segments into a $3 \mathrm{D}$ distribution is represented with two 2D plots (figures 3(B) and (C)). The elements of the 3D structure are summed along the third mode obtaining a 2D distribution plot. For example, by summing along the mode $m_{2}$ we generate a $2 \mathrm{D}$ distribution:

$$
\mathbf{X} 2\left(m_{1}, m_{3}\right)=\sum_{i=1}^{10} \underline{\mathbf{X}}\left(m_{1}, i, m_{3}\right) \text {. }
$$

\subsection{Creating a tensor distribution structure}

For every segment within a cEEG epoch we calculate quantized $m_{1}, m_{2}$ and $m_{3}$ index values. Next, the corresponding three-dimensional structure is created by counting the prevalence of EEG segments with a particular feature distribution $\underline{\mathbf{X}}\left(m_{1}, m_{2}, m_{3}\right)$. We illustrate in figures $4-6$ that the generated 3D distribution structures have clearly distinguishable patterns for different background EEG grades. For each class (mild, medium and severe), 3D distribution structures are averaged across all $1 \mathrm{~h}$ graded cEEG epochs and depicted in figures 4-6.

\subsection{Tensor decomposition techniques: dimensionality reduction step}

The main motivation to use multi-dimensional structures is to extract discriminative and interpretable information. The created 3D structure is represented as a 3-way tensor $\mathbf{X}$ of size $\mathfrak{R}^{9 \times 10 \times 12}$, where each element characterizes corresponding multi-channel EEG activity. Due to a large number of histogram frequency bins $(9 \times 10 \times 12=1080)$, classical supervised classification techniques cannot be directly applied. The size of the training and testing datasets are too small to obtain a reliable classification due to limited number of encephalopatic neonates $(<50)$. Therefore, to reduce the model dimensions, a recently developed tensor decomposition technique is applied to extract a new, discriminative reduced set of features from a tensor $\underline{\mathbf{X}}$. The method is based on a Tucker $N$-way decomposition model. For three-dimensional data, the basic Tucker3 model of a tensor $\underline{\mathbf{X}}$ is illustrated in figure 7 .

The Tucker- $N$ decomposition model is represented as a product of a core tensor $\underline{\mathbf{G}}$ and a set of factor (basis) matrices $\mathbf{U}^{(n)}$ :

$$
\underline{\mathbf{X}}=\underline{\mathbf{G}} \times_{1} \quad \mathbf{U}^{(1)} \times_{2} \quad \mathbf{U}^{(2)} \times_{3} \mathbf{U}^{(3)}+\underline{\mathbf{E}} .
$$

Here, the mode- $n$ product $\mathbf{G} \times{ }_{n} \mathbf{U}^{(n)}$ of a core tensor $\mathbf{G}$ and a matrix $\mathbf{U}^{(n)}$ is a tensor product of a tensor and a matrix along the mode $n$ :

$$
\begin{aligned}
& \underline{\mathbf{X}}=\underline{\mathbf{G}} \times_{n} \mathbf{U}^{(n)}, x_{i_{1}, i_{2}, \ldots, i_{n-1}, i_{n}, i_{n+1}, \ldots, i_{N}}=\sum_{j_{n}=1}^{J_{n}} g_{j_{1}, j_{2}, \ldots, j_{N}} u_{i_{n}, j_{n}}, \\
& \underline{\mathbf{G}} \in \mathfrak{R}^{J_{1} x J_{2} x \ldots J_{N}}, \quad \mathbf{U} \in \mathfrak{R}^{I_{n} x J_{n}}, \underline{\mathbf{X}} \in \mathfrak{R}^{I_{1} x \ldots I_{n-1} x I_{n} x I_{n+1} x \ldots x I_{N}},
\end{aligned}
$$

The core tensor $\mathbf{G}$ is usually of much smaller dimensions than the original tensor $\mathbf{X}$ and tensor $\mathbf{E}$ characterizes the approximation error of a model (or fit). In case that factor matrices and a core tensor are orthogonal, the Tucker model can be considered as an extension of the singular value decomposition (SVD) model, known as the higher order SVD (HOSVD) (De Lathauwer et al 2000). To calculate factor basis $\mathbf{U}^{(n)}$, different algorithms can be applied with respect to the constraints imposed on the factor basis. For instance, orthogonality can be imposed leading to the well-known higher order orthogonal iterations algorithm (De Lathauwer et al 2000). In addition, non-negativity, sparseness or statistical mutual independence of the factor basis can be imposed (Phan and Cichocki 2010). In this work, we will apply constraints to create a core tensor with discriminative properties that is useful for the multi-class classification tasks (Phan and Cichocki 2010). That is, class labels are incorporated into the algorithm to better capture and model the differences of input data tensors $(\underline{\mathbf{X}})$ between data classes. The applied algorithm, higher order discriminant analysis (HODA), represents the generalization of the 1D linear discriminant analysis to the multi-linear one (Phan and Cichocki 2010, Phan 2011). Mathematical details of the HODA algorithm and how it is applied onto EEG data are described in appendix A. 
A

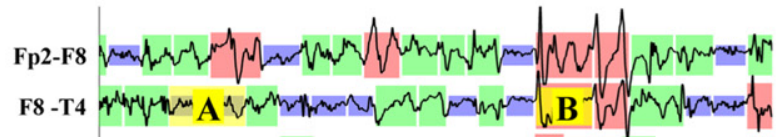

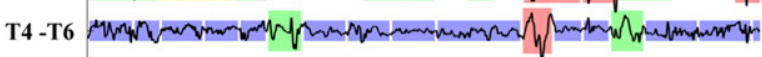

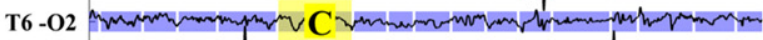

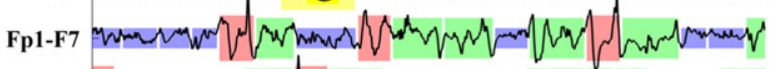

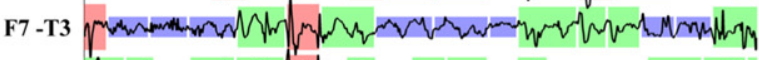

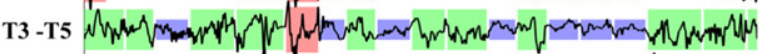

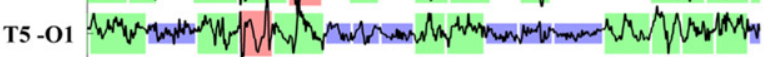

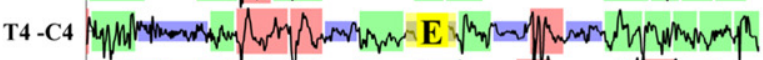

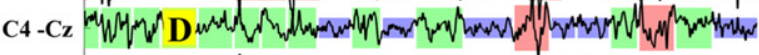

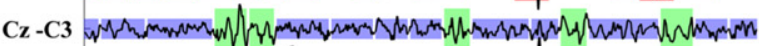

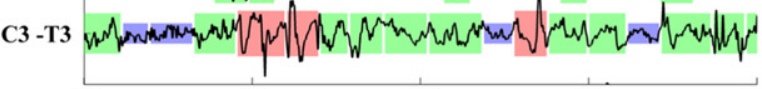

\begin{tabular}{ccccc}
\hline 5 & 20 & 25 & 30 & 35 \\
& & time $[s]$ & &
\end{tabular}

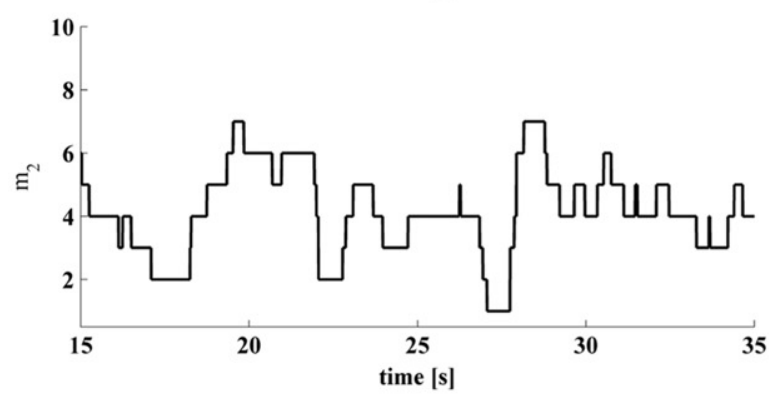

B
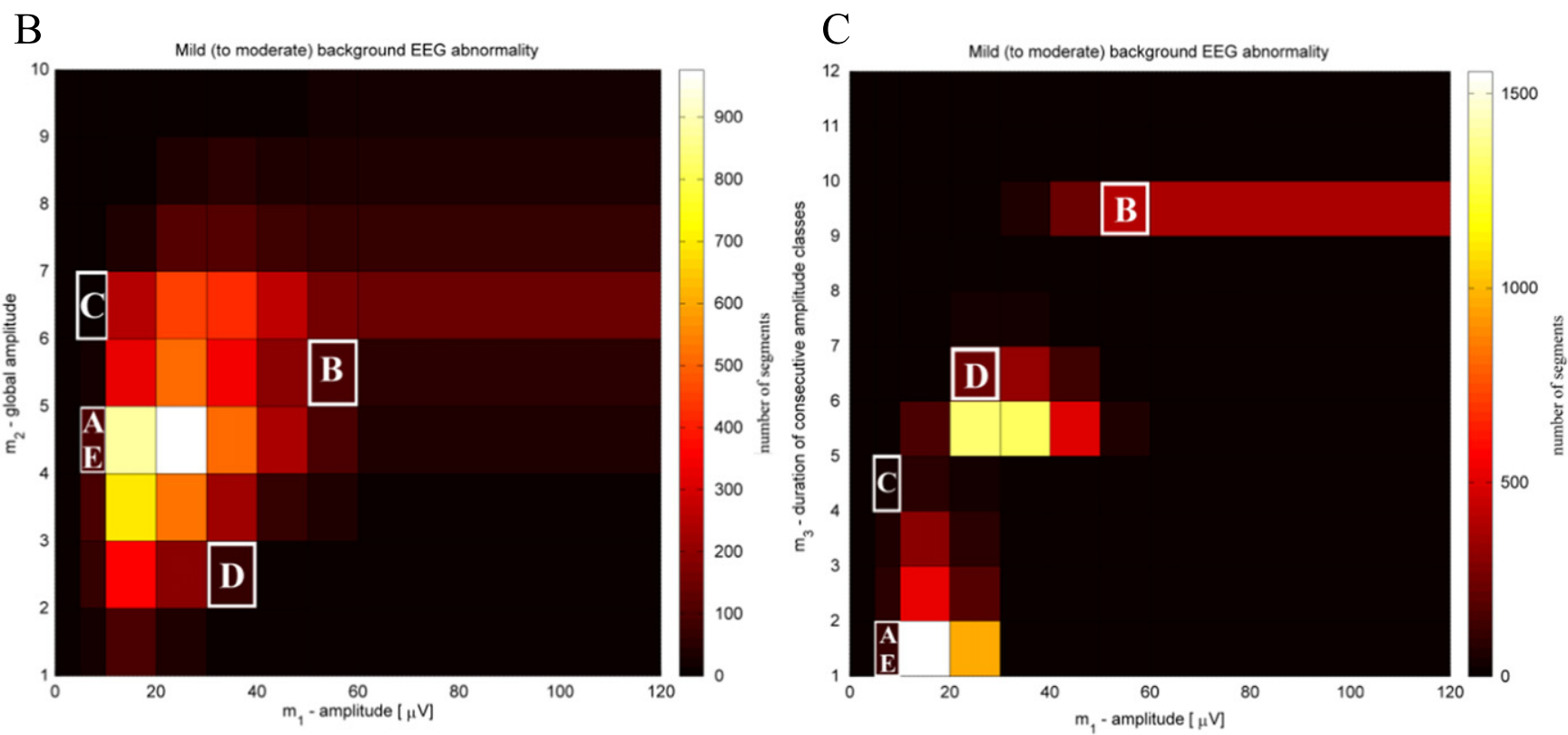

Figure 3. (A) Epoch of EEG signal is adaptively segmented. The segments are colored according to their amplitude values into blue (Low), green (Medium) and red (High). Five segments are marked to illustrate the quantification of various interplays with the adjacent segments: A $(2,4,1) \mathrm{B}(7,5,9), \mathrm{C}(2,6,4) \mathrm{D}(4,2,6), \mathrm{E}(2,4,1)$. In addition, feature index values $m_{2}$ representing global amplitude are displayed. (B) Mapping of the EEG segments from (A) into the distribution structure. The view is displayed along the axis $m_{1}$ and $m_{2}$, whereas the color bar represents the number of EEG segments in a particular frequency bin. (C) Mapping of the EEG segments from (A) into the distribution structure. The view is displayed along the axis $m_{1}$ and $m_{3}$, whereas the color bar represents the number of EEG segments in a particular frequency bin. 

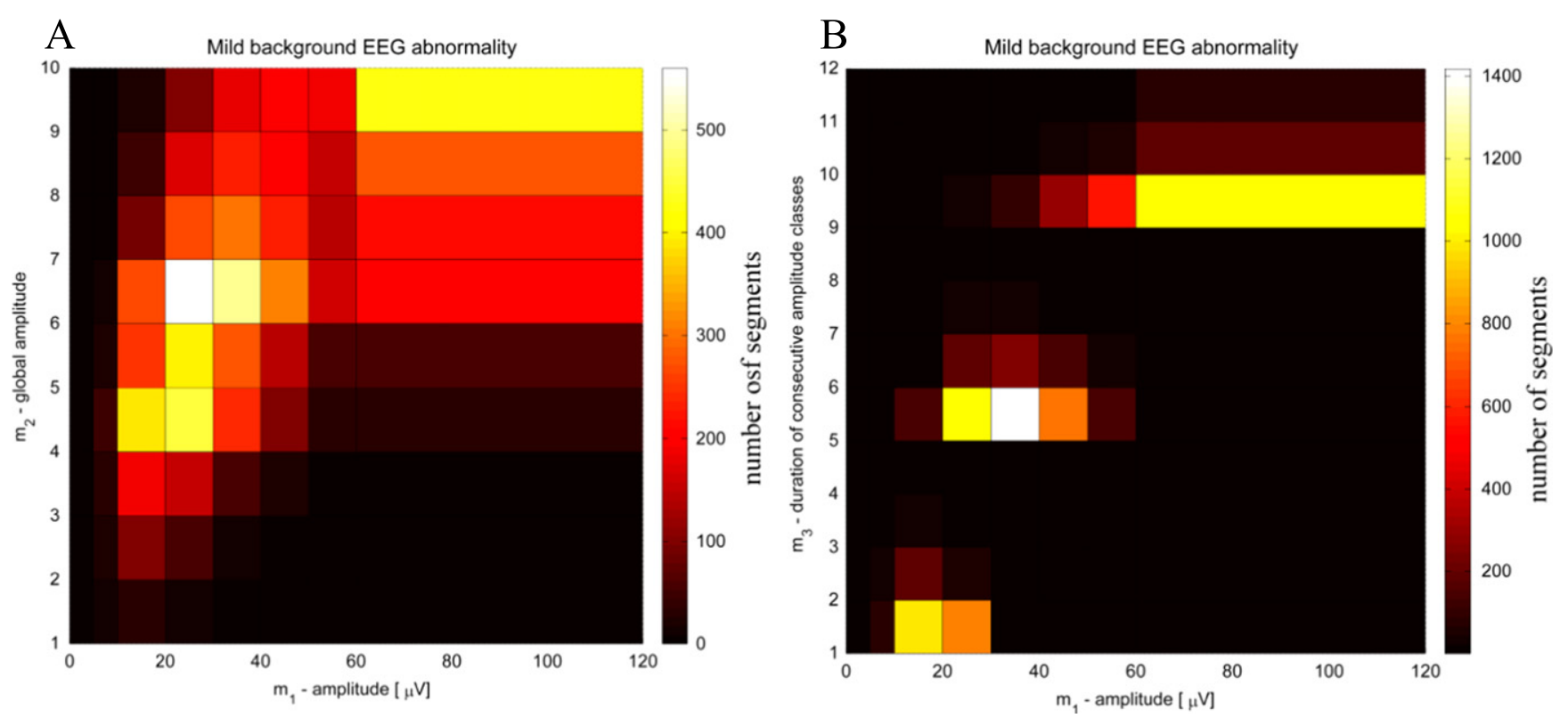

Figure 4. (A) 2D distribution of mild background EEG abnormalities displayed along modes $m_{1}$ and $m_{2}$, whereas the color bar represents the number of EEG segments in a particular frequency bin. (B) 2D distribution of mild background EEG abnormalities displayed along modes $m_{1}$ and $m_{3}$, whereas the color bar represents the number of EEG segments in a particular frequency bin.
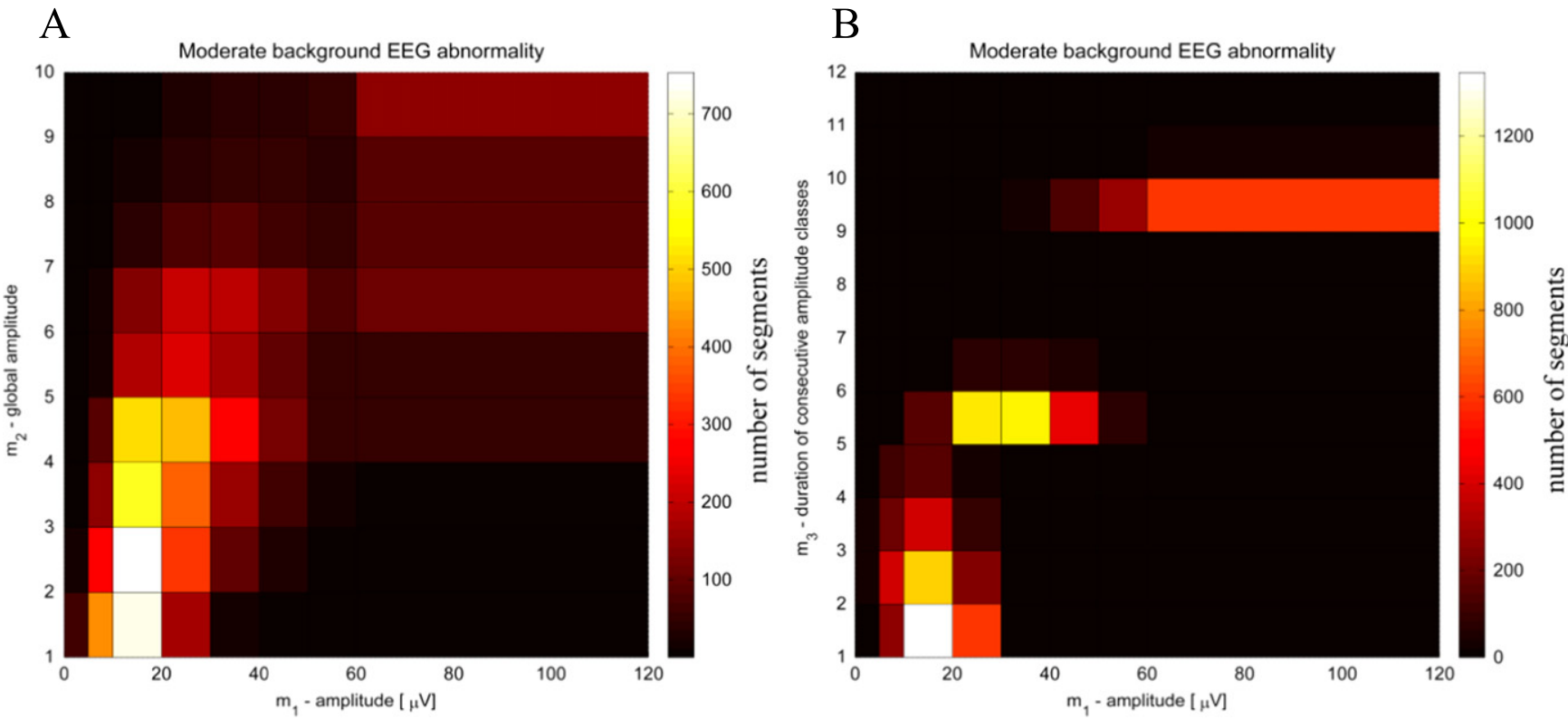

Figure 5. (A) 2D distribution of moderate background EEG abnormalities displayed along modes $m_{1}$ and $m_{2}$, whereas the color bar represents the number of EEG segments in a particular frequency bin. (B) 2D distribution of moderate background EEG abnormalities displayed along modes $m_{1}$ and $m_{3}$, whereas the color bar represents the number of EEG segments in a particular frequency bin.

In summary, the HODA algorithm tries to optimize the factor basis in such a way that the core tensors $\underline{\mathbf{G}}$ from the same classes are as similar as possible. On the other hand, it aims to increase the 'differences' of the core tensors from the different classes. The training part of the algorithm calculates the TUCKER bases using the training data set (figure 8).

As the next step, we use the estimated factor basis $\mathbf{U}^{(n)}$ with a HODA algorithm to build a projection filter (figure 8). To extract the set of features from the test data tensors $\underline{\mathbf{X}}^{(t)}$ (class labels are not known) we create the corresponding core tensors:

$$
\stackrel{\circ}{\mathbf{G}}^{(t)}=\underline{\stackrel{X}{\mathbf{X}}}^{(t)} \times_{1} \mathbf{U}^{(1) \mathrm{T}} \times_{2} \mathbf{U}^{(2) \mathrm{T}} \times_{3} \mathbf{U}^{(3) \mathrm{T}} .
$$

That is, the test data tensors are projected onto a feature subspace spanned by the calculated set of basis factors $\mathbf{U}^{(n)}$. In addition, since the calculated factor basis $\mathbf{U}^{(n)}$ are orthogonal, according to the HODA algorithm implementation, the regular transpose operator of the matrix, ${ }^{\mathrm{T}}$ is applied.

The extracted features, elements of the core tensor $\underline{\mathbf{G}}^{(t)}$, are then compared with the training features using a 

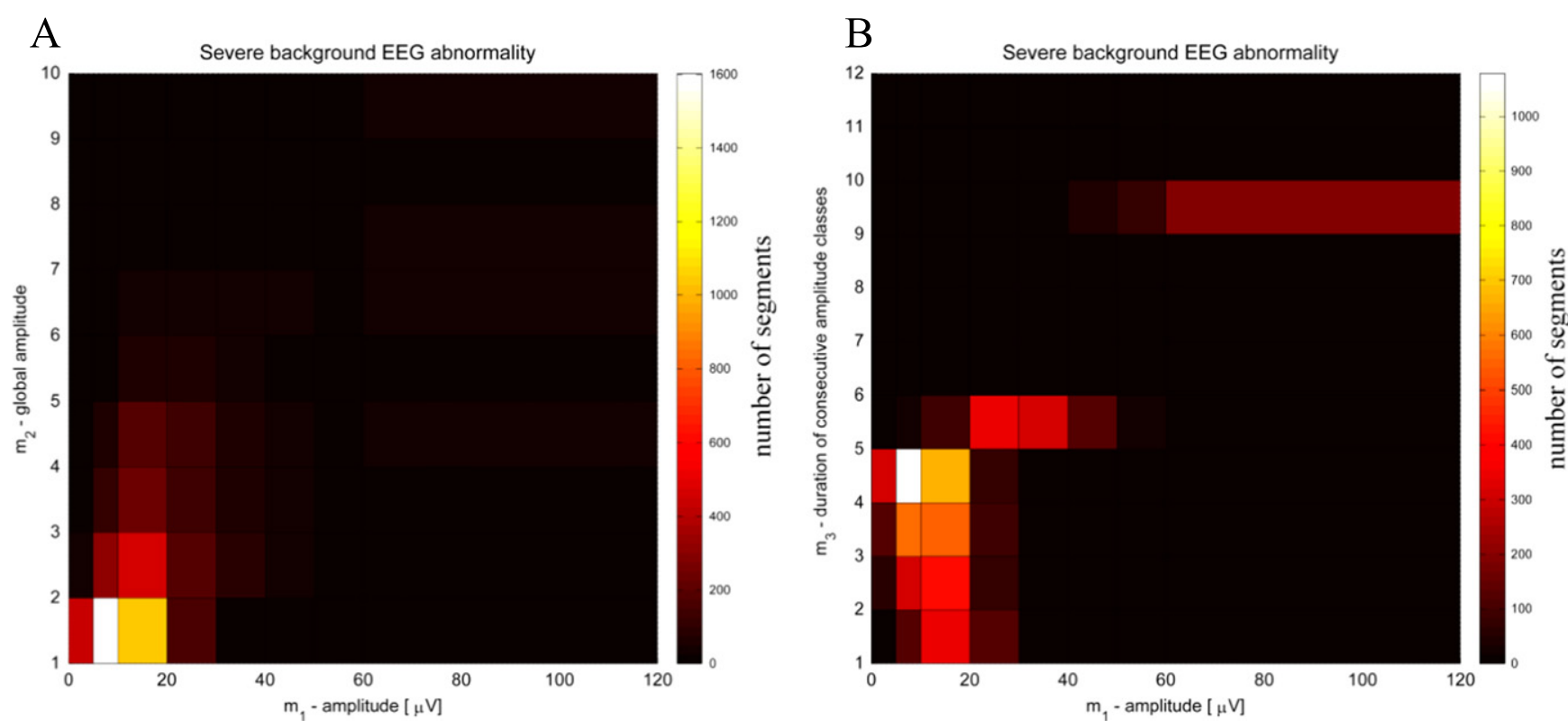

Figure 6. (A) 2D distribution of severe background EEG abnormalities displayed along modes $m_{1}$ and $m_{2}$, whereas the color bar represents the number of EEG segments in a particular frequency bin. (B) 2D distribution of severe background EEG abnormalities displayed along modes $m_{1}$ and $m_{3}$, whereas the color bar represents the number of EEG segments in a particular frequency bin.
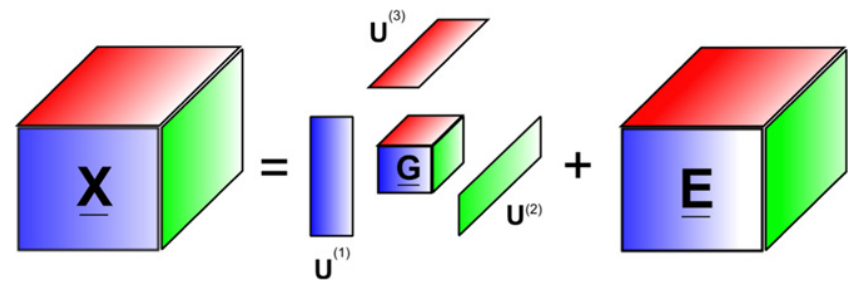

Figure 7. Illustration of a tensor decomposition using a Tucker-3 model. The objective is to estimate factor matrices $\mathbf{U}^{(n)}$ and a core tensor $\mathbf{G}$. In general, the constraints are imposed on factor matrices $\mathbf{U}^{(n)}$ such as orthogonality, non-negativity and/or statistical mutual independence. If the orthogonality is imposed, the decomposition can be seen as an extension of the singular value decomposition (SVD) into a higher dimension.

supervised machine learning classifier (least squares support vector machine (LS-SVM)). The training and test phase of the algorithm and the overall classification scheme are depicted in figure 8 .

\subsection{Classification}

After the decomposition step, the reduced set of discriminative features $(\underline{\mathbf{G}})$ is calculated both for training and test data samples. To accomplish this, HODA algorithm's parameters were set to model (fit) $95 \%$ of the original data tensor. In this way, the original data tensors $(9 \times 10 \times 12)$ are decomposed, resulting in core tensors $(\mathbf{G})$ of dimensions $4 \times 4 \times 5(=80)$. The number of 80 features is still high and a subset of the most significant and discriminative features (elements) from the core tensors are selected as proposed in (Phan and Cichocki 2010). Fisher information ranking score has been applied during the training (and testing) phase and the information indices are calculated for all elements from the core tensors $\mathbf{G}$ and are sorted into descending order. Further classification has been performed using 25 most discriminative elements and used as input feature vectors into LS-SVM classifiers (figure 8).

Next, standard inductive supervised classification techniques are applied to classify corresponding background EEG grades. In this study, the LS-SVM classifier has been applied (Suykens et al 1999). To perform multi-class classification (3 classes), prior encoding of the classes is applied, resulting in training of two LS-SVM classifiers using Gaussian kernel. A 'one_versus_one' encoding scheme has been applied as proposed in (Pelckmans et al 2002). Next, a four-fold crossvalidation method has been applied and as a criterion function 'misclassification error' has been used. Additionally, the leave-one patient out (LOO) method has been applied during training and testing phase. For a single test patient, 8 epochs of $1 \mathrm{~h}$ cEEG were used for the automated grading, whereas the $2641 \mathrm{~h}$ cEEG epochs were used for the training phase. During each iteration the decomposition of the input data tensors and the training of the LS-SVM classifiers have been repeated.

\section{Results}

\subsection{Automated assessment of background EEG of $1 \mathrm{~h}$ long epochs}

The results of the background EEG classification are presented as a confusion matrix in table 3 . The table offers insight into an agreement of automated classification compared to visual background EEG grading by an experienced clinical neurophysiologist (PJC). The achieved classification accuracy is $89 \%$. The algorithm successfully classified the 


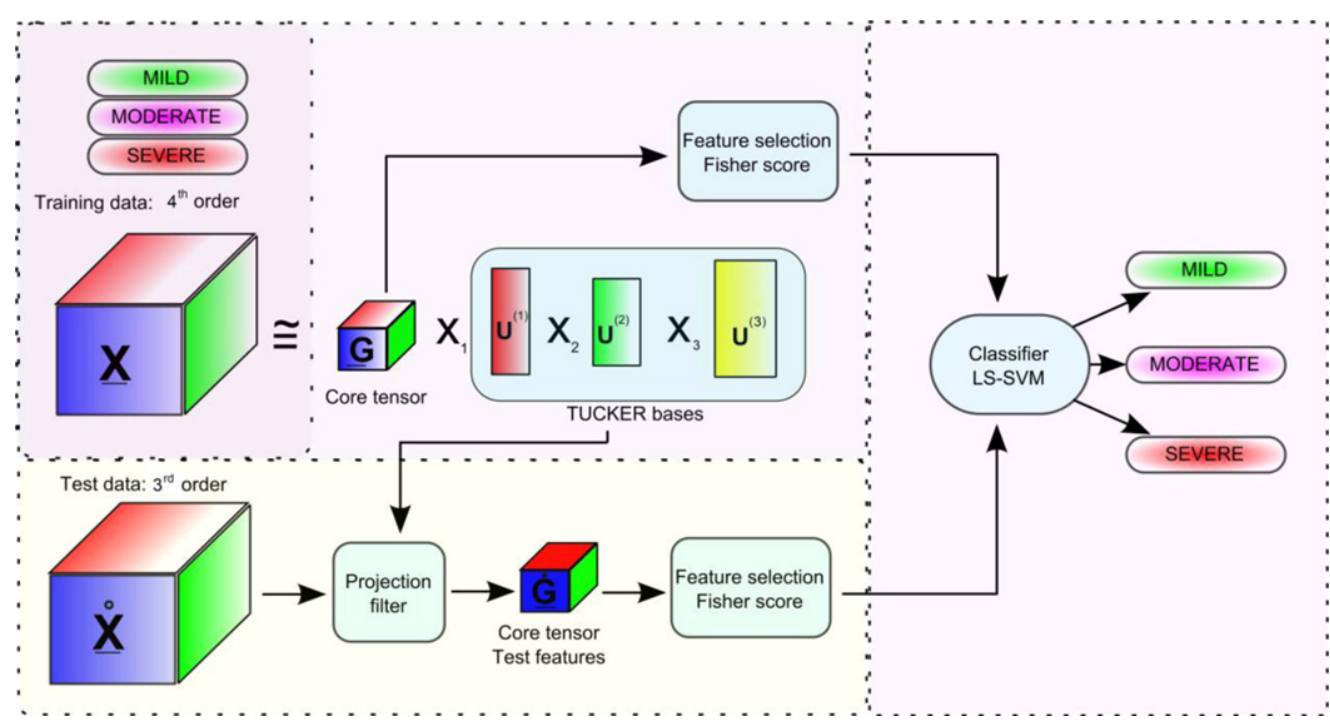

Figure 8. Flow chart illustrating a classification procedure based on the Tucker decomposition of the concatenated tensor $\underline{\mathbf{X}}$ consisting of all sampling training data $\underline{\mathbf{X}}^{(k)}$. Reduced features are obtained by projecting the testing data tensor $\underline{\dot{X}}$ onto the feature subspace spanned by factors (bases) $\mathbf{U}^{(n)}$ (projection filter). The most discriminative elements are selected from the core tensors, using the Fischer selection score and used for the final classification. Output of the least-squares support vector machine (LS-SVM) classifiers provides the assessed background EEG grade.

Table 3. Confusion matrix represents agreement of automated classification method and the expert EEG reader. $1 \mathrm{~h}$ cEEG epochs were classified into mild, moderate or severe background EEG class. All classified epochs represent non-preselected, real case EEG recordings. The overall classification accuracy of $89 \%$ (= $(73+44+126) / 272)$ has been achieved.

\begin{tabular}{llll}
\hline $\begin{array}{l}\text { Automated } \begin{array}{l}\text { reader } \\
\text { reat EEG }\end{array} \\
\text { Mild }\end{array}$ & MILD & MODERATE & SEVERE \\
\hline $\begin{array}{l}\text { Moderate } \\
\text { Severe }\end{array}$ & 7 & $61 \%)$ & 1 \\
Achieved accuracy & 0 & 8 & 7 \\
\hline
\end{tabular}

majority of severe and mild cases. Moderate background EEG class resulted in a slightly higher number of misclassifications, as it is the 'in-between' class between mild and severe. In 31 patients, correct grading of $1 \mathrm{~h}$ cEEG epochs ranged from 6 to 8 out of 8 epochs. In two patients, EEG grades were 'in-between' mild and moderate resulting in 4 correct detections out of 8 epochs. Finally, for one neonate, showing severe background EEG with very pronounced artifacts, 4 epochs were misclassified.

\subsection{Benchmarking}

To additionally confirm the results, we examine the scores determined by an expert EEG reader and the automated classifier. We portray those grades with the median IBI values detected within the $1 \mathrm{~h}$ of cEEG using the automated IBI detector (Matic et al 2012). As can be seen from figure 9, the grades are correlated with the median IBI values. Severe background EEG can be determined using IBI detector as it mainly consists of burst-suppression patterns. On the other

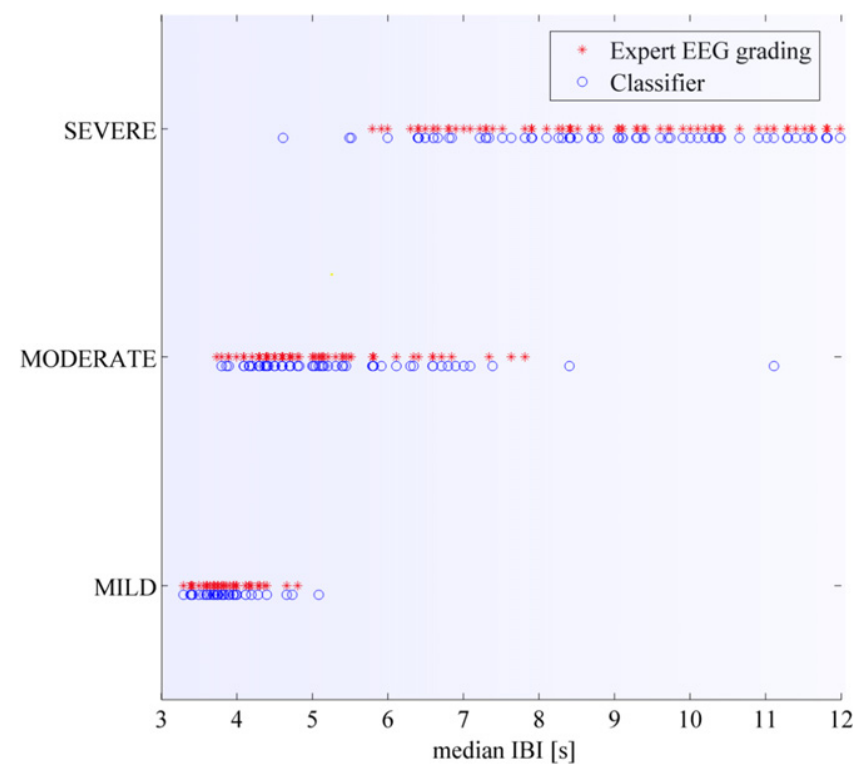

Figure 9. Comparison of background EEG grades scored on $1 \mathrm{~h}$ of cEEG by an experienced clinical neurophysiologist (red) and automated assessment of those grades (blue). These grades show correlation with the median interburst interval (IBI) values. Background EEG activity not containing IBIs are not displayed here (40 out of 80 mild (to normal) background EEG grades).

hand, mild and moderate grades do not always have easily interpretable but rather overlapping ranges of IBIs demonstrating the added value of the new framework. Additionally, 40 out of $801 \mathrm{~h}$ cEEG epochs (50\%) scored as grade mild do not contain IBI patterns that can be used for background EEG parameterization. In such cases, our approach still allows to parameterize EEG segments as it does not rely on the strict concept of IBI. 


\section{Discussion}

This work was motivated by the need to improve the automated EEG analysis in the NICUs and in particular to assist in real-time monitoring of neonates with HIE. Here, we presented a novel method for the automated assessment of background EEG abnormalities. It is based on a new concept that uses intrinsic structure of 3D prevalence of the designed segments' features. The method classifies cEEG periods of $1 \mathrm{~h}$ into mild, moderate or severe background EEG classes. First, the method exploits the concept of adaptive EEG segmentation and processing of segments' features in the segments' feature space (Barlow 1985, Krajca et al 1991). We suggested to quantify the obtained segments with three features, which to some degree mimic the visual interpretation of the background EEG abnormalities. Next, we discretized the segments' feature space and represented it as a large 3-way tensor. In this way, continuous EEG data streams have been parameterized. The obtained data tensors have then been efficiently decomposed using a recently developed, HODA algorithm (Phan and Cichocki 2010). In this way, a small discriminative set of features has been extracted from the data tensor. Next, using LS-SVM classifiers high accuracy has been achieved to classify background EEG abnormalities. In addition, we did not preselect data based on absence of artifacts and seizures, effectively demonstrating that the proposed method is robust enough to be used in clinical practice.

The main original contribution of this study is the effective and holistic EEG parameterization step. In general, this is a challenging task as features have to be extracted from multiple EEG channels from $1 \mathrm{~h}$ duration of recordings. Our approach of EEG quantification is an extension of the concepts initially proposed in (Barlow 1985). In that study, adaptive EEG segments, were mapped into the segments' feature space and certain EEG patterns were explored. In another study, researchers adaptively segmented the EEG signal and used segments' features, expressed as median values, to quantify EEG data in newborns (Paul et al 2003). Here, instead of a single feature's median value, we used a high-dimensional histogram to better capture the overall segments' feature distribution. For instance, we designed nine frequency bins to describe overall amplitude distribution within EEG data. In this way, more discriminative EEG parameterization is obtained, thereby quantifying the differences between distributions' heavy tails. Quantifying these differences, a more effective modeling of background EEG classes has been accomplished. We have extensively tested other segments' features such as power frequency bands (alpha, beta, theta, delta), duration of segments as well as some of the implemented 'hand-chosen' features. All these features were calculated independently for a single EEG segment as done in the previous studies (Barlow 1985, Paul et al 2003, 2006), but did not give great discrimination between classes. Here, two new features were found more effective to consolidate temporal and spatial relations between adjacent EEG segments $\left(m_{2}\right.$ and $\left.m_{3}\right)$. In this way, we succeeded to capture and exploit the multi-channel structure of the EEG signal.
During visual assessment of background EEG abnormalities, researchers define EEG discontinuity and suppression of amplitude as the main hallmark for the EEG quantification (Watanabe et al 1980, Murray et al 2009, Tsuchida et al 2013). More specifically, classical 'burst-suppression' IBIs and/or tracé discontinue IBIs are used for the visual classification of background EEG severity. The guiding rules for background EEG scoring use duration of IBIs as follows: mild $(\mathrm{IBI}<5 \mathrm{~s})$, moderate $(\mathrm{IBI}<10 \mathrm{~s})$ and severe $(10 \mathrm{~s}<\mathrm{IBI}<60 \mathrm{~s})$ (Murray et al 2009, Cherian et al 2011). With respect to this, duration of IBIs is indirectly quantified into the $3 \mathrm{D}$ distribution tensor. In particular, low amplitude segments were used to detect IBIs in our previous studies (Matic et al 2012, 2014). Therefore, longer duration of consecutive low amplitude classes $\left(m_{3} \in[2-4]\right)$ which are also globally attenuated across all EEG channels $\left(m_{2} \in[1-2]\right)$ correspond to IBI patterns. For instance, in moderate background EEG abnormalities duration of IBIs is assumed to be less than $10 \mathrm{~s}$. Therefore, EEG segments constituting IBIs $<10 \mathrm{~s}$ would be mapped into a tensor area where EEG is globally attenuated $\left(m_{2} \in[1-2]\right)$ and where duration of consecutive low amplitude segments is less than $10 \mathrm{~s}$ $\left(m_{3} \in[1-2]\right)$. In contrast, in severe background EEG abnormalities $(10 \mathrm{~s}<$ IBIs $<60 \mathrm{~s})$, the duration of consecutive low amplitude classes would be longer than $10 \mathrm{~s}$ having $m_{3} \in[3-4]$. Therefore, tensor structure can effectively discriminate various trends of IBI duration encountered in neonatal EEG data.

Background EEG of neonates with severe HIE consists of mainly burst-suppression patterns (Flisberg et al 2011, Walsh et al 2011). These background EEG states can be quantified with an automated IBI detection method (Löfhede et al 2008, Matic et al 2012). However, in mild and moderate HIE, EEG background consists of periods of more continuous background activity, expressing higher amplitude variability. Hence, EEG quantification using only IBI values would not be possible (Biagioni et al 1999). Because of this, researchers defined various EEG states and patterns such as 'transitory discontinuous' (Pezzani et al 1986), 'modified' burst-suppression (Sinclair et al 1999) and 'constantly discontinuous' (Biagioni et al 1999). In such cases, an expert EEG reader attempts to assess background EEG grades according to the level of amplitude suppression, variability and global amplitude attenuations. Consequently, this motivated us to structure these characteristics into a distribution tensor where we distinguish subtle differences in amplitude values, global level of suppression and alternations of three amplitude classes $(L, M$ and $H$ ). It is important to note that, although various background EEG scoring systems (and terminology) have been used, grading using a single system is usually rather consistent with very high inter-rater agreement and consensus (Cherian et al 2011, Korotchikova et al 2011). Last but not least, HIE injury is not static and it evolves over time. When a neonate is recovering from severe asphyxia, EEG is becoming more continuous and burst suppression patterns evolve into tracé discontinue and further to tracé alternant patterns. In such cases, the method developed for IBI detection would stop working accurately. On the other hand, our method will 
provide accurate background EEG assessment during all phases of monitoring.

In the literature, other approaches for the background EEG quantification are based on the combination of qEEG features that were fed into supervised classifiers (Löfhede et al 2010, Korotchikova et al 2011, Stevenson et al 2013). In these studies, the EEG features were calculated on fixed-size windows (e.g. $1 \mathrm{~min}$ ), and were further averaged across EEG channels and across time interval (e.g. $1 \mathrm{~h}$ ). In this way, the researchers assume that HIE is expressed in EEG as a global phenomenon. However, the assumption that HIE is global as observed in EEG does not necessarily always hold. In particular, it is the case in milder EEG asymmetries that the set of features' values averaged across all EEG channels would be difficult to discriminate. On the other hand, our approach investigates segments' amplitude distribution within single EEG channels and can detect subtle EEG abnormalities. For instance, severe attenuation of $40 \%$ of the EEG channels implies localized brain injuries (e.g. frontal, occipital areas). This would be reflected in a tensor representation since large number of prolonged consecutive low amplitude classes would occur (e.g. $m_{3} \in[2-4]$ ), and yet there would be no global EEG attenuation (e.g. $m_{2} \in[3-5]$ ). Another advantage of the tensorial framework is that it can easily be expanded into a 4D tensor, where mode-4 would correspond to unequivocal brain areas: frontal, parietal, central and occipital. Then, the method would be able to explicitly explain milder EEG abnormalities. This would assist to better study the brain functioning in an automated way. Naturally, other features (modes) can be added into a model, such as a recently developed 'activation synchrony index', which would incorporate interhemispheric synchrony (Räsänen et al 2013).

In addition, our method proved to be very robust to major artifacts. For instance, transient high amplitude EEG patterns (e.g. movement, electrode and eye artifacts and/or paroxysmal like activity-seizures) would be mainly mapped into 'frequency bins' with very high amplitudes (e.g. $>60 \mu \mathrm{V}$ and $\left.m_{1} \in[8-9]\right)$. Hence, they would not interfere with the properties of the remaining EEG segments of prior interest (e.g. $<60 \mu \mathrm{V})$ and further background EEG classification. Other patterns, such as for instance bursts, would be mapped into globally 'high' amplitude frequency bins and would be analyzed separately from suppressed intervals. On the other hand, approaches that use fixed-length windows would not assess burst and suppression patterns independently, but those would be averaged across proposed qEEG features.

Moreover, an interpretation of the applied features is of paramount importance. Measures related to spectral entropy (Löfhede et al 2010) or white Gaussian processes (Stevenson et al 2013) are not intuitive and easily interpretable. On the other hand, a parameterized distribution model is both discriminative and can be used for visual interpretation. This is a distinctive advantage of our method as we can interpret a cEEG epoch $(1 \mathrm{~h})$ with two compact 2D distribution plots. Moreover, the distribution plots provide information which are consistent with the visual grading rules as defined in (Murray et al 2009). For instance, the proposed amplitude values of mild background EEG abnormalities (e.g. range $30-50 \mu \mathrm{V}$ ) can be observed in 2D distribution plots.

In this study, we have applied tensor methods to structure and process the parameterized EEG data. On the other hand, in previous studies researchers commonly used vectors to quantify and classify continuous EEG data. For instance, the classification method for the detection of 'burst-suppression' background EEG state applied feature vectors and the classification accuracy was high (Löfhede et al 2010). Yet, this approach performed poorly during the classification of more subtle sleep states in newborns. Compared to this approach, more effective parameterization of EEG sleep states has been achieved in (Paul et al 2006) and, as a result, it yielded a large number of parameterization elements structured as a matrix. Subsequently, the comparisons between sleep states (quiet versus active sleep) have been obtained with factor analysis. In our study we parameterized EEG with a much larger number of elements and employed more advanced methods based on multi-linear algebra that can manage high-dimensional data. The proposed tensor decomposition methods have been successfully applied in many neuroscientific researches and represent a new concept in brain data analysis (Cichocki 2013).

The classification accuracy of background EEG classes obtained in our study is $89 \%$. Therefore, it is similar as the results achieved in (Stevenson et al 2013) where a value of $83 \%$ is reported. However, these results were obtained on preselected data where parts with major artifacts were excluded and the epochs were inspected so that the background EEG grade is consistent throughout the $1 \mathrm{~h}$ cEEG epoch. Therefore, in the real case scenarios, it can be concluded that the accuracy of the proposed methods would decrease. On the other hand, our data contained major artifacts as well as epileptic seizures expressing compelling seizure burden in 18 neonates. Moreover, the data have been selected randomly and background EEG grade within $1 \mathrm{~h}$ was not always consistent.

In this study, the presence of long-lasting severe artifacts mostly affected the EEG data classification. Therefore, the future research studies should be aimed towards the development of even more robust methods and the validation of methods should be performed on large datasets without the pre-selection of the data. In such cases, two directions are feasible, (1) to develop a method which is very robust to artifacts or (2) to develop a method which would pre-select EEG data. For the latter, EEG data periods severely affected with artifacts can be excluded using approaches similar to one proposed in (e.g. Wulsin et al 2011, Nowotny et al 2013). Consequently, the method should provide an automated output for clean EEG periods, assisting in this way clinical staff in the NICUs.

\section{Acknowledgments}

We thank our patients and their parents for their co-operation and the neurotechnologists who made the high quality EEG recordings. The methods developed are part of a future, non- 
commercial bed-side automated brain monitoring system (http://www.innovatienetwerk.be/projects/1791 and https:// neoguard.net) for supporting clinicians in assessing the severity of brain dysfunction and its eventual recovery in neonatal encephalopathy. VM, NK and SVH are supported by: Research Council KUL: GOA/10/09 MaNet, CoE PFV/ 10/002 (OPTEC); Flemish Government: FWO: projects: G.0427.10N (Integrated EEG-fMRI), G.0108.11 (Compressed Sensing) G.0869.12N (Tumor imaging) G.0A5513N (Deep brain stimulation); IWT: projects: TBM 080658-MRI (EEG-fMRI), TBM 110697-NeoGuard; iMinds Medical Information Technologies SBO 2014, ICON: NXT_Sleep; Flanders Care: Demonstratieproject Tele-Rehab III (2012-2014); Belgian Federal Science Policy Office: IUAP P7/19/ (DYSCO, 'Dynamical systems, control and optimization', 2012-2017); Belgiuan Foreign Affairs-Development Cooperation: VLIR UOS programs; EU: the research leading to these results has received funding from the European Research Council under the European Union's Seventh Framework Programme (FP7/2007-2013)/ERC Advanced Grant: BIOTENSORS ( $\mathrm{n}^{\circ}$ 339804). This paper reflects only the authors' views and the Union is not liable for any use that may be made of the contained information; other EU funding: RECAP 209G within INTERREG IVB NWE programme, EU MC ITN TRANSACT 2012 ( $n^{\circ}$ 316679), ERASMUS EQR: Community service engineer ( ${ }^{\circ}$ 539642-LLP-1-2013).

\section{Appendix A.}

\section{HODA approach for multi-way features}

Consider an available set of multidimensional tensors $\underline{\mathbf{X}}^{(k)} \in \mathfrak{R}^{I_{1} \times I_{2} \times I_{3}}, \quad(k=1,2, . ., K)$, representing a training data belonging to $C$ classes. Each training sample $\underline{\mathbf{X}}^{(k)}$ is given a label $c_{k}$ indicating the category (class) to which it belongs. In our problem label $c_{k}$ represents the class (degree) of background EEG abnormality as scored visually by an expert EEG reader. To perform a dimensionality reduction and to extract significant features, we apply simultaneous approximate tensor decompositions (figure A1):

$\underline{\mathbf{X}}^{(k)}=\underline{\mathbf{G}}^{(k)} \times_{1} \mathbf{U}^{(1)} \times_{2} \mathbf{U}^{(2)} \times_{3} \mathbf{U}^{(3)}+\underline{\mathbf{E}}, \quad k=1,2, \ldots, K$, where the compressed core tensors $\underline{\mathbf{G}}^{(k)} \in \mathfrak{R}^{J_{1} x J_{2} x \cdot J_{3}}$ are of lower dimension than the original data tensors $\underline{\mathbf{X}}^{(k)}$. Here, we assume that the factors (basis matrices) $\underline{\mathbf{U}}^{(n)} \in \mathfrak{R}^{I_{n} x J_{n}}$ are the same for all training data tensors $(k=1,2, . ., K)$.

The simultaneous approximate tensor decomposition problem is equivalent to performing a Tucker- $N$ decomposition of a tensor obtained by concatenating all $N$-way data tensors into a tensor of order $N+1$ (Phan and Cichocki 2010). In other words, to compute the factor matrices $\mathbf{U}^{(n)}$ and the core tensors $\underline{\mathbf{G}}^{(k)}$, we concatenate all training tensors into one fourth order training data tensor $\underline{\mathbf{X}}=\operatorname{cat}\left(\underline{\mathbf{X}}^{(1)}, \underline{\mathbf{X}}^{(2)}, \ldots, \underline{\mathbf{X}}^{(K)}, N\right.$ +1 ), with $N+1=4$. Next, we perform the Tucker-3
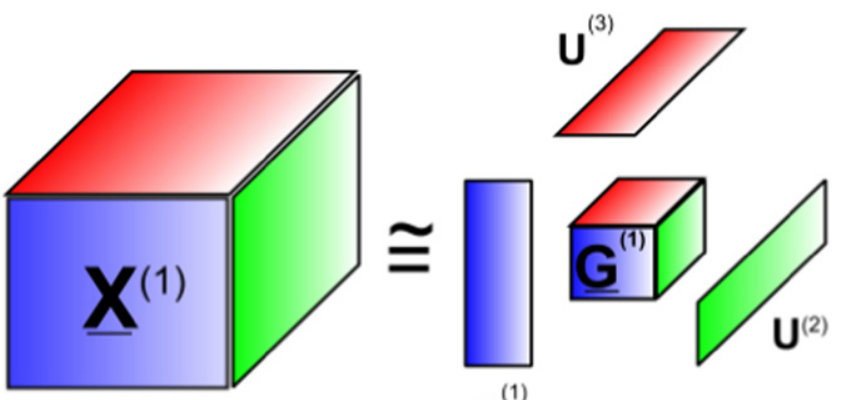

$\mathbf{U}^{(1)}$
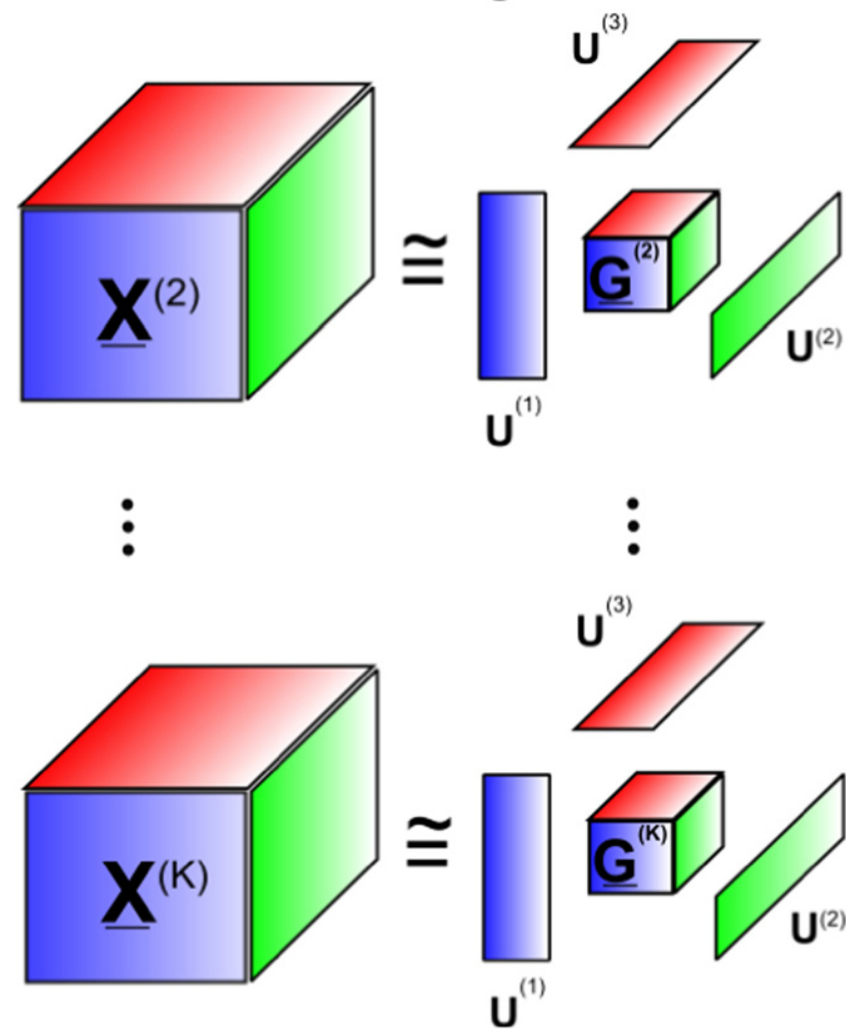

Figure A1. Illustration of feature extraction from a set of 3-way data tensors $\underline{\mathbf{X}}^{(k)}, k=1,2, \ldots, K$. The objective is to estimate common factor matrices (basis) $\mathbf{U}^{(n)}, n=1,2,3$ and the core tensors $\underline{\mathbf{G}}^{(k)}$.

decomposition:

$$
\underline{\mathbf{X}}=\underline{\mathbf{G}} \times \times_{1} \mathbf{U}^{(1)} \times_{2} \quad \mathbf{U}^{(2)} \times_{3} \mathbf{U}^{(3)}+\underline{\mathbf{E}} .
$$

The sample tensors $\underline{\mathbf{X}}^{(k)}$ can be extracted back from the concatenated tensors by fixing the fourth index at a value $k$, i.e. $\underline{\mathbf{X}}(:,:,:, k)=\underline{\mathbf{X}}^{(k)}$ and the individual features (corresponding to different classes) are extracted from the core tensor $\underline{\mathbf{G}} \in \mathfrak{R}^{J_{1} x J_{2} x . J_{3} x J_{4}}$ as $\underline{\mathbf{G}}^{(k)}=\underline{\mathbf{G}}(:,:,:, \ldots, \ldots)$, with $J_{4}=k$. In other words, the features of a specific training data $\underline{\mathbf{X}}^{(k)}$ are represented by the $k$ th row of the mode- 4 matricized version of the core tensor $\underline{\mathbf{G}}$.

Furthermore, the problem needs to estimate the basis matrices $\underline{\mathbf{U}}^{(n)}$ for the concatenated tensor of all training data $\underline{\mathbf{X}}^{(k)}$ incorporating the known labels (class membership) as an $\bar{a}$ priori information. This represents an algorithm, HODA (Phan and Cichocki 2010). In general, the HODA algorithm finds the orthogonal basis $\mathbf{U}^{(n)}$ based on the maximization of 
the Fisher ratio between the core tensors $\underline{\mathbf{G}}^{(k)}$ :

$$
\phi=\underset{\mathbf{U}^{(1)}, \ldots, \mathbf{U}^{(N)}}{\operatorname{argmax}} \frac{\sum_{c=1}^{C} K_{c}\left\|\underline{\mathbf{G}}^{(c)}-\overline{\overline{\mathbf{G}}}\right\|_{F}^{2}}{\sum_{k=1}^{K}\left\|\underline{\mathbf{G}}^{(k)}-\underline{\overline{\mathbf{G}}}^{\left(c_{k}\right)}\right\|_{F}^{2}} .
$$

Here, $\underline{\overline{\mathbf{G}}}^{(c)}$ is the mean tensor of the $c$ th class consisting of $K_{c}$ training samples, $c_{k}$ denotes the class to which the $k$ th training sample $\underline{\mathbf{X}}^{(k)}$ belongs and $\underline{\overline{\mathbf{G}}}$ is the mean tensor of all training samples.

\section{References}

Barlow J 1985 Computer characterization of tracé alternant and REM sleep patterns in the neonatal EEG by adaptive segmentation-an exploratory study Electroencephalogr. Clin. Neurophysiol. 60 163-73

Barlow J, Creutzfeldt O, Michael D, Houchin J and Epelbaum H 1981 Automatic adaptive segmentation of clinical EEGs Electroencephalogr. Clin. Neurophysiol. 51 512-25

Biagioni E, Bartalena L, Boldrini A, Pieri R and Cioni G 1999 Constantly discontinuous EEG patterns in full-term neonates with hypoxic-ischaemic encephalopathy Clin. Neurophysiol. $1101510-5$

Bodenstein G, Schneider W and Malsburg C 1985 Computerized EEG pattern classification by adaptive segmentation and probability-density-function classification. Description of the method Comput. Biol. Med. 15 297-313

Cherian P, Deburchgraeve W, Swarte R, De Vos M, Govaert P, Van Huffel S and Visser G 2011 Validation of a new automated neonatal seizure detection system: a clinician's perspective Clin. Neurophysiol. 122 1490-9

Cichocki A 2013 Tensor decompositions: a new concept in brain data analysis? (arXiv:1305.0395)

Creutzfeldt O, Bodenstein G and Barlow J 1985 Computerized EEG pattern classification by adaptive segmentation and probability density function classification. Clinical evaluation Electroencephalogr. Clin. Neurophysiol. 60 373-93

Deburchgraeve W, Cherian P, De Vos M, Swarte R, Blok J, Visser G, Govaert P and Van Huffel S 2008 Automated neonatal seizure detection mimicking a human observer reading EEG Clin. Neurophysiol. 119 2447-54

De Lathauwer L, De Moor B, V and Vandewalle J 2000 A multilinear singular value decomposition SIAM J. Matrix Anal. Appl. 21 1253-78

Despotovic I et al 2013 Relationship of EEG sources of neonatal seizures to acute perinatal brain lesions seen on MRI: a pilot study Hum. Brain Mapp. 34 2402-17

De Vos M, Deburchgraeve W, Cherian P, Matic V, Swarte R, Govaert P, Visser G and Van Huffel S 2011 Automated artifact removal as preprocessing refines neonatal seizure detection Clin. Neurophysiol. 122 2345-54

De Vos M, Riés S, Vanderperren K, Vanrumste B, Alario F, Huffel V and Burle B 2010 Removal of muscle artifacts from EEG recordings of spoken language production Neuroinformatics 8 135-50

De Vos M, Vergult A, De Lathauwer L, De Clercq W, Van Huffel S, Dupont P, Palmini A and Van Paesschen W 2007 Canonical decomposition of ictal scalp EEG reliably detects the seizure onset zone NeuroImage 37 844-54

De Vries L and Hellström-Westas L 2005 Role of cerebral function monitoring in the newborn Arch. Disease Child.-Fetal Neonatal Edn. 90 201-7
Flisberg A, Kjellmer I, L'ofhede J, Lindecrantz K and Thordstein M 2011 Prognostic capacity of automated quantification of suppression time in the EEG of post-asphyctic full-term neonates Acta Paediatrica 100 1338-43

Gerla V, Paul K, Lhotska L and Krajca V 2009 Multivariate analysis of full-term neonatal polysomnographic data IEEE Trans. Inf. Technol. Biomed. 13 104-10

Gotman J, Flanagan D, Zhang J and Rosenblatt B 1997 Automatic seizure detection in the newborn: methods and initial evaluation Electroencephalogr. Clin. Neurophysiol. 103 356-62

Hagberg B, Hagberg G, Beckung E and Uvebrant P 2001 Changing panorama of cerebral palsy in Sweden: part 8. Prevalence and origin in the birth year period 1991-94 Acta Paediatrica 90 271-7

Jennekens W, Ruijs L, Lommen C, Niemarkt H, Pasman J, van Kranen-Mastenbroek V, Wijn P, van Pul C and Andriessen P 2011 Automatic burst detection for the EEG of the preterm infant Physiol. Meas. 321623

Joyce C, Gorodnitsky I and Kutas M 2004 Automatic removal of eye movement and blink artifacts from EEG data using blind component separation Psychophysiology 41 313-25

Kinoti S 1993 Asphyxia of the newborn in east, central and southern Africa East Afr. Med. J. 70 422-33

Klemm M, Haueisen J and Ivanova G 2009 Independent component analysis: comparison of algorithms for the investigation of surface electrical brain activity Med. Biol. Eng. Comput. 47 413-23

Koolen N, Jansen K, Vervisch J, Matic V, De Vos M, Naulaers G and Van Huffel S 2014 Line length as a robust method to detect high-activity events: automated burst detection in premature EEG recordings Clin. Neurophysiol. doi:10.1016/j.clinph.2014.02.015

Korotchikova I, Stevenson N, Walsh B, Murray D and Boylan G 2011 Quantitative EEG analysis in neonatal hypoxic ischaemic encephalopathy Clin. Neurophysiol. 122 1671-8

Krajca V, Petránek S, Patáková I and Värri A 1991 Automatic identification of significant graphoelements in multichannel EEG recordings by adaptive segmentation and fuzzy clustering Int. J. Biomed. Comput. 28 71-89

Löfhede J, Löfgren N, Thordstein M, Flisberg A, Kjellmer I and Lindecrantz K 2008 Classification of burst and suppression in the neonatal electroencephalogram J. Neural Eng. 5402

Löfhede J, Thordstein M, Löfgren N, Flisberg A, Rosa-Zurera M, Kjellmer I and Lindecrantz K 2010 Automatic classification of background EEG activity in healthy and sick neonates J. Neural Eng. 7016007

Matic V, Cherian P, Jansen K, Koolen N, Naulaers G, Swarte R, Govaert P, Visser G, Van Huffel S and De Vos M 2012 Automated EEG inter-burst interval detection in neonates with mild to moderate postasphyxial encephalopathy IEEE EMBC $17-20$

Matic V, Cherian P, Jansen K, Koolen N, Naulaers G, Swarte R, Govaert P, Visser G, Van Huffel S and De Vos M 2014 Automated detection of dynamic interburst intervals in the EEG of neonates with hypoxic ischemic encephalopathy Clin. Neurophysiol. submitted

Mitchell T, Neil J, Zempel J, Thio L, Inder T and Bretthorst G 2013 Automating the analysis of EEG recordings from prematurelyborn infants: a bayesian approach Clin. Neurophysiol. 124 452-61

Murray D, Boylan G, Ryan C and Connolly S 2009 Early EEG findings in hypoxic-ischemic encephalopathy predict outcomes at 2 years Pediatrics 124 459-67

Nowotny T, Rospars J, Martinez D, Elbanna S and Anton S 2013 Machine learning for automatic prediction of the quality of electrophysiological recordings PloS One 880838 
Palmu K, Wikström S, Hippeläinen E, Boylan G, Hellström-Westas L and Vanhatalo S 2010 Detection of 'EEG bursts' in the early preterm EEG: visual versus automated detection Clin. Neurophysiol. 121 1015-22

Paul K, Krajca V, Roth Z, Melichar J and Petranek S 2003 Comparison of quantitative EEG characteristics of quiet and active sleep in newborns Sleep Med. 4 543-52

Paul K, Krajca V, Roth Z, Melichar J and Petranek S 2006 Quantitative topographic differentiation of the neonatal EEG Clin. Neurophysiol. 117 2050-8

Pelckmans K, Suykens J, Van Gestel T, De Brabanter J, Lukas L, Hamers B, De Moor B and Vandewalle J 2002 LS-SVMlab: a matlab/c toolbox for least squares support vector machines Tutorial. KULeuven-ESAT (Leuven, Belgium)

Pezzani C, Radvanyi-Bouvet M F, Relier J P and Monod N 1986 Neonatal electroencephalography during the first twenty-four hours of life in full-term newborn infants Neuropediatrics 17 $11-8$

Phan 2011 Tensor toolbox for feature extraction and applications Matlab Software Toolbox (www.bsp.brain.riken.jp/ $/ \mathrm{phan} / \mathrm{nfea} /$ nfea.html)

Phan A and Cichocki A 2010 Tensor decompositions for feature extraction and classification of high dimensional datasets Nonlinear Theory Appl., IEICE 1 37-68

Räsänen O, Metsäranta M and Vanhatalo S 2013 Development of a novel robust measure for interhemispheric synchrony in the neonatal EEG: activation synchrony index (ASI) NeuroImage $69256-66$

Richardus J, Graafmans W, Verloove-Vanhorick S and Mackenbach J 2003 Differences in perinatal mortality and suboptimal care between ten European regions: results of an international audit BJOG: Int. J. Obstetrics Gynaecology 110 97-105

Scher M 2004 Automated EEG-sleep analyses and neonatal neurointensive care Sleep Med. 5 533-40
Sinclair D, Campbell M, Byrne P, Prasertsom W and Robertson C 1999 EEG and long-term outcome of term infants with neonatal hypoxic-ischemic encephalopathy Clin. Neurophysiol. 110 655-9

Stevenson N, Korotchikova I, Temko A, Lightbody G, Marnane W and Boylan G 2013 An automated system for grading EEG abnormality in term neonates with hypoxicischaemic encephalopathy Ann. Biomed. Eng. 41 775-85

Suykens J and Vandewalle J 1999 Least squares support vector machine classifiers Neural Process. Lett. 9 293-300

Temko A, Thomas E, Marnane W, Lightbody G and Boylan G 2011 EEG-based neonatal seizure detection with support vector machines Clin. Neurophysiol. 122 464-73

Tsuchida T et al 2013 American clinical neurophysiology society standardized EEG terminology and categorization for the description of continuous EEG monitoring in neonates: report of the american clinical neurophysiology society critical care monitoring committee J. Clin. Neurophysiol. 30 161-73

Vanderperren K et al 2013 Single trial ERP reading based on parallel factor analysis Psychophysiology 50 97-110

Van Hese P, Philips W, De Koninck J, Van de Walle R and Lemahieu I 2001 Automatic detection of sleep stages using the EEG 3rd IEEE/EMBS Spec. Top. Conf. Microtechnol. Med. Biol. 2 1944-7

Walsh B, Murray D and Boylan G 2011 The use of conventional EEG for the assessment of hypoxic ischaemic encephalopathy in the newborn: a review Clin. Neurophysiol. 122 1284-94

Watanabe K, Miyazaki S, Hara K and Hakamada S 1980 Behavioral state cycles, background EEGs and prognosis of newborns with perinatal hypoxia Electroencephalogr. Clin. Neurophysiol. 49 618-25

Wulsin D, Gupta J, Mani R, Blanco J and Litt B 2011 Modeling electroencephalography waveforms with semi-supervised deep belief nets: fast classification and anomaly measurement J. Neural Eng. 8036015 